\title{
Plasma Exchange for the Treatment of Transient Extreme Hypertriglyceridemia Associated with Diabetic Ketoacidosis and Acute Pancreatitis
}

\author{
Davide Donelli ${ }^{1}$, Lorenzo Morini ${ }^{1}$, Chiara Trenti $^{1}$, Rosaria Santi $^{1}$, Dimitriy Arioli ${ }^{2}$, Emanuele Alberto Negri $^{1}$ \\ ${ }^{1}$ Alta Intensità Medica, Department of Internal Medicine, Arcispedale Santa Maria Nuova - IRCCS, Reggio Emilia, Italy \\ ${ }^{2}$ Medicina II Cardiovascolare, Department of Internal Medicine, Arcispedale Santa Maria Nuova - IRCCS, Reggio Emilia, Italy
}

\section{Doi: 10.12890/2018_000853-European Journal of Case Reports in Internal Medicine - @ EFIM 2018}

\begin{abstract}
Received: $23 / 01 / 2018$
Accepted: $16 / 02 / 2018$
\end{abstract}

Published: 08/03/2018

How to cite this article: Donelli D, Morini L, Trenti C, Santi R, Arioli D, Negri EA. Plasma exchange for the treatment of transient extreme
hypertriglyceridemia associated with diabetic ketoacidosis and acute pancreatitis. EJCRIM 2018;5: doi:10.12890/2018_000853.

Conflicts of Interests: The Authors declare that there are no competing interests.

This article is licensed under a Commons Attribution Non-Commercial 4.0 License

\section{ABSTRACT}

Diabetic ketoacidosis (DKA) can quite frequently present in association with acute pancreatitis (AP) caused by transient severe hypertriglyceridemia (HTG). Here we report the case of a patient presenting with DKA, severe HTG and AP who received urgent plasma exchange for HTG control, and who reached adequate serum triglyceride levels only after appropriate DKA management. The treatment of patients presenting with DKA and coexistent AP associated with severe HTG should focus first on appropriate DKA management. Plasma exchange as a treatment for severe HTG in patients with DKA and AP should be evaluated carefully.

\section{LEARNING POINTS}

- The treatment of patients presenting with diabetic ketoacidosis, acute pancreatitis and severe hypertriglyceridemia should focus first on diabetic ketoacidosis management.

- Plasma exchange as a treatment for severe hypertriglyceridemia in patients with diabetic ketoacidosis and acute pancreatitis should be evaluated carefully.

- Triglyceride concentrations should always be measured in case of diabetic ketoacidosis.

\section{KEYWORDS}

Diabetic ketoacidosis, acute pancreatitis, hypertriglyceridemia, plasma exchange.

\section{INTRODUCTION}

Diabeticketoacidosis(DKA) canquiteoften presentinassociation withacutepancreatitis(AP) caused by transientseverehypertriglyceridemia (HTG) which is determined by DKA metabolic dysregulation ${ }^{[1]}$. Patients presenting with this particular triad of DKA, severe HTG and AP can be challenging since they must be appropriately managed. Here we report the case of a patient presenting with DKA, severe HTG and AP who received urgent plasma exchange for HTG control, and who reached adequate serum triglyceride levels only after appropriate DKA management. 


\section{CASE REPORT}

A 37-year-old Chinese man was admitted to the emergency department for epigastric pain.

At admission the patient's laboratory values were: $\mathrm{pH} 7.29$ (7.32-7.42), $\mathrm{HCO}_{3}-17.8 \mathrm{mmol} / \mathrm{l}(24.0-28.0)$, lactate $11 \mathrm{mg} / \mathrm{dl}(4.00-14.00)$, glucose $357 \mathrm{mg} / \mathrm{dl}$ (65-110), bilirubin $2.9 \mathrm{mg} / \mathrm{dl}$ (0.2-1.0), anion gap $13 \mathrm{mEq} / \mathrm{l}, \mathrm{WBC} 13.1 \times 1,000 / \mathrm{ml}$ (4.0-11.0) and Hb 17.2 g/dl (13.5-17.5). Other laboratory values were not measured since blood samples were lipemic. The patient did not report a history of diabetes mellitus but had experienced a recent $15 \mathrm{~kg}$ weight loss with polyuria and polydipsia. Therefore, an initial diagnosis of DKA was made (blood ketones were later reported to be $2.0 \mathrm{mmol} / \mathrm{l}$ ) and insulin administration was started.

An abdominal CT scan also revealed severe AP with ascites and retroperitoneal oedema. Serum amylase and lipase levels were normal. Serum triglyceride levels (after dilution) were above $7,000 \mathrm{mg} / \mathrm{dl}$ and, since severe HTG was interpreted as the cause of AP, independently from the concurrent DKA, urgent plasma exchange was performed. Plasma exchange was considered urgent in order to stop the pathogenesis of AP, while overlooking the fact that DKA might have been the primary cause determining the extreme HTG. The procedure took place the next morning, after which serum triglycerides stabilized at $980 \mathrm{mg} / \mathrm{dl}$.

The patient was transferred in the afternoon to our step-down unit, but without appropriate insulin treatment, showed uncompensated acidosis (pH 7.31), elevated blood ketones $(2.3 \mathrm{mmol} / \mathrm{l})$, elevated blood glucose $(224 \mathrm{mg} / \mathrm{dl})$ and dehydration. Consequently, the patient started appropriate i.v. insulin administration, with glycemic measurement every hour and blood ketone measurement every 6 hours, according to our treatment algorithm for DKA. He also received generous fluid replacement, and a nasojejunal feeding tube was placed for early refeeding in anticipation of AP treatment once DKA had resolved. The patient refused analgesia.

Interestingly, after 16 hours DKA had completely resolved with normalization of $\mathrm{pH}$, blood ketones, anion gap, $\mathrm{HCO}_{3}-$ and serum triglycerides, which had dropped to $260 \mathrm{mg} / \mathrm{dl}$.

Further HBA1c measurement $(117 \mathrm{mmol} / \mathrm{ml}, 12.7 \%)$ confirmed unknown pre-existing diabetes mellitus.

\section{DISCUSSION}

Rarely, diabetic coma can be a complication of severe AP in patients with no history of diabetes, but more often DKA has been described as the primary disorder that leads to $\mathrm{AP}^{[1,2]}$. DKA has also been described as inducing $\mathrm{HTG}$, since insulin deficit leads to lipolysis and peripheral lipoprotein lipase inhibition, thus causing an increase in triglycerides ${ }^{[2]}$.

Severe HTG with serum triglyceride levels above $1,000 \mathrm{mg} / \mathrm{dl}$ can cause $A \mathrm{P}^{[3]}$, and hypertriglyceridemic AP is thought to be caused by the action of pancreatic lipase, which hydrolyzes excess triglycerides with subsequent accumulation of noxious free fatty acids in the pancreas ${ }^{[3]}$. Moreover, a study showed that during DKA severe transient HTG is a pathogenetic factor for AP ${ }^{[2,3]}$ and that in such cases severe transient HTG tends to resolve with appropriate DKA management ${ }^{[2]}$.

Plasma exchange has been described as a treatment option for hypertriglyceridemic pancreatitis ${ }^{[3]}$ and should be considered in patients who have severe HTG independently of other factors. However, when extreme HTG and subsequent AP are associated with hyperglycemia, adequate glycemic control should be the first therapeutic option ${ }^{[3]}$, because, as discussed above, with DKA resolution the HTG tends to nearly normalize ${ }^{[2]}$.

In the described case, the patient received urgent plasma exchange in order to lower serum lipids but did not actually achieve safe serum triglyceride levels after the procedure. Therefore, the plasma exchange treatment was not 'life-saving' and did not effectively remove the AP pathogenetic factor.

Moreover, the patient did not receive immediate adequate treatment for DKA, which presumably was the primary disorder due to an unknown pre-existing diabetes mellitus. A similar patient should have been treated adequately for his DKA from the very beginning as the main priority.

In fact, in line with the evidence ${ }^{[2]}$, after appropriate DKA management was started, HTG readily resolved with DKA resolution, which suggests that for the management of patients presenting with DKA, AP and severe HTG, the focus should first be on appropriate DKA treatment. Moreover, in the described case priority given to glycemic control would also have been in line with optimal management for hypertriglyceridemic $A P^{[3]}$. Furthermore, as discussed above, DKA is a separate risk factor for severe HTG.

All these considerations raise questions about the role of plasma exchange for the treatment of HTG with coexisting DKA and AP. Plasma exchange is an expensive procedure, and if simpler treatment based on appropriate management of DKA could resolve the severe HTG in an acceptable time frame, then it should be preferred.

An interesting question is why some patients develop severe HTG (>1,000 mg/dl) during DKA. Genetic susceptibilities may enhance serum triglycerides elevation as a study ${ }^{[4]}$ showed that, especially in the Chinese population, a CFTR mutation and a TNF promoter polymorphism are associated with hyperlipidemic pancreatitis. 


\section{CONCLUSIONS}

The treatment of patients presenting with DKA and coexisting AP associated with severe HTG should focus first on appropriate DKA management in order to also lower serum triglyceride concentrations to safe levels. Prompt resolution of DKA is also central to allow early enteral feeding for the treatment of AP. Plasma exchange as a treatment for severe HTG in patients with DKA and AP should be evaluated carefully since it is very expensive and may not be as effective as simpler DKA management. Moreover, in case of DKA, attention should always be given to the measurement of serum triglycerides, and an abdominal CT scan for potential AP should be performed when DKA presents with compatible abdominal pain.

\section{REFERENCES}

1. Nair S. Diabetic ketoacidosis, hyperlipidemia, and acute pancreatitis: the enigmatic triangle. Am J Gastroenterol 1997;92:1560-1561.

2. Nair S, Yadav D, Pitchumoni CS. Association of diabetic ketoacidosis and acute pancreatitis: observations in 100 consecutive episodes of DKA. Am J Gastroenterol 2000;95:27952800.

3. Tsuang W, Navaneethan U, Ruiz L, Palascak JB, Gelrud A. Hypertriglyceridemic pancreatitis: presentation and management. Am J Gastroenterol 2009;104:984-991.

4. Chang YT, Chang MC, Su TC, Liang PC, Su YN, Kuo CH, et al. Association of cystic fibrosis transmembrane conductance regulator (CFTR) mutation/variant/haplotype and tumor necrosis factor (TNF) promoter polymorphism in hyperlipidemic pancreatitis. Clin Chem 2008;54:131-138. 